\title{
Medizinische Grenzentscheidungen
}

\section{Yvonne Gilli}

Dr. med., Mitglied des FMH-Zentralvorstandes, Departementsverantwortliche Digitalisierung/eHealth

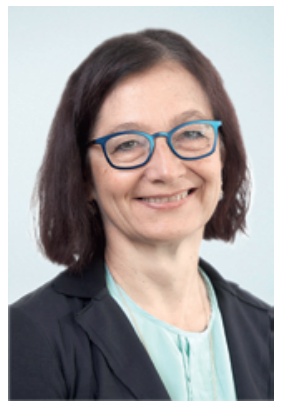

Medizinische Grenzentscheidungen stellen die Ärztin und den Arzt vor besondere ethische Herausforderungen. Spontan denken wir an Entscheidungen in akuten lebensbedrohlichen Situationen oder am Lebensende. Grenzentscheidungen können auch Menschen betreffen, die besonders vulnerabel sind, weil sie situationsbedingt nicht eigenverantwortlich über die ihnen zustehende Behandlung bestimmen können. Zu ihnen gehören Asylsuchende, die unser Land wegen eines rechtskräftigen behördlichen Wegweisungsentscheids verlassen müssen.

Die menschliche Würde ist unantastbar. Die Schweiz als Rechtsstaat respektiert die damit verbundenen Grundrechte. Dazu gehört das Recht auf eine medizinische Grundversorgung, sofern Hinweise auf eine Gesundheitsgefährdung bestehen oder die betroffene Person eine solche geltend macht. In der Regel ist es die Gefängnisärztin, der Gefängnisarzt, welcher für die medizinische Einschätzung verantwortlich ist. Sie/er stützt sich in der Beurteilung neben dem Fachwissen auf die standesethischen Richtlinien. Bereits 2013 hat der damalige Präsident der Zentralen Ethikkommission der Schweizerischen Akademie der Medizinischen Wissenschaften auf die problematische Praxis der ärztlichen Versorgung bei zwangsweisen Rückführungen hingewiesen und Verbesserungen gefordert [1]. Insbesondere kritisierte er die ungenügende Regelung der Aufgaben und Verantwortlichkeiten und wies darauf hin, dass Gefängnisärztinnen und -ärzte

\section{In einem Pilotprojekt wurden}

die Aufgaben und Verantwortlichkeiten von Gefängnisärzten bei der zwangsweisen Rückführung neu definiert.

meistens vom Justiz- und Polizeidepartement angestellt sind und somit einen Loyalitätskonflikt haben. In ihrer Arbeit sind sie der ärztlichen Kunst verpflichtet. Gleichzeitig stehen sie in einem Abhängigkeitsverhältnis zum Arbeitgeber, welcher die Rückführung angeordnet hat.

In der Zwischenzeit wurden in einem Pilotprojekt Verbesserungen erprobt, unter Einbezug aller betroffenen Kreise. Neu erstellen die Gefängnisärztinnen und -ärzte einen strukturierten vertraulichen Bericht, in dem sie erkennbare Gefährdungen der Gesundheit festhalten und prüfen, ob gemäss internationalen Richtlinien Kontraindikationen vorliegen für die Flugtauglichkeit. Die Transportfähigkeit selbst müssen sie nicht beurteilen. Diese fällt in die Zuständigkeit derjenigen Organisation, welche die Rückführungen organisiert und dafür eine eigene ärztliche Expertise bereitstellt. Der Austausch der medizinischen Informationen zwischen den beteiligten Ärztinnen und Ärzten wird sichergestellt mit der schriftlichen Erlaubnis der rückzuführenden Person. Die medizinische Begleitung der Rückführung ist gewährleistet, und auf medizinische Zwangsmassnahmen wird verzichtet.

Ärztliche Beurteilungen von Menschen, welche unser Land unfreiwillig verlassen, bleiben Grenzentscheidungen.

Die ärztliche Betreuung im Kontext von Wegweisungsentscheiden und behördlichen Zwangsmassnahmen bleibt schwierig. Gefängnisärztinnen und -ärzte bleiben Angestellte derjenigen Behörde, welche für den Wegweisungsentscheid verantwortlich ist. Sie stehen im Spannungsfeld rechtskräftiger Entscheide und einer ärztlichen Ethik, welche die Gesundheit der Patientin und des Patienten ins Zentrum stellt. Das Wohlergehen ist aber nicht nur abhängig vom aktuellen ärztlichen Bericht, sondern auch von der zukünftigen medizinischen Versorgung im Ausland. Wir alle wissen, dass den meisten abgewiesenen Personen nach ihrer Rückführung keine gesicherte medizinische Versorgung mehr zur Verfügung steht. Allein schon die Beschaffung wirksamer Medikamente wird für viele eine tägliche Herausforderung, für einige unmöglich. In diesem Sinn bleiben ärztliche Beurteilungen von Menschen, welche unser Land unfreiwillig verlassen, Grenzentscheidungen. Sowohl die Verantwortungsträger in der Gesellschaft als auch die Ärzteschaft sind verpflichtet, die Rahmenbedingungen zu reflektieren. Individuell ist es immer auch ein Gewissensentscheid der verantwortlichen Ärztin / des verantwortlichen Arztes, wo sie/er die Grenze zieht zwischen ethischer Verantwortung und ihrer/seiner Rolle gegenüber rechtsstaatlichen Entscheiden. 